\title{
Diseño de un curso autogestivo modular en línea de metodología de la investigación para universitarios Design of a self-managed modular and online research methodology course for university students
}

\author{
Guarneros-Reyes, Esperanza ${ }^{1}$; Espinoza-Zepeda, Argenis Josué2; Silva Rodríguez, Arturo $^{3}$ y \\ Sánchez-Sordo, José Manuel ${ }^{4}$ \\ Universidad Nacional Autónoma de México
}

\section{RESUMEN}

El objetivo de este trabajo fue diseñar un curso de metodología de la investigación, que sea autogestivo modular en línea, dirigido a estudiantes universitarios, como también describir la ruta de aprendizaje de los estudiantes. Para diseñar este curso formado por siete módulos, se siguió el modelo de e-curso basado en la experiencia. En el proceso se obtuvo una estructura del curso autogestivo y modular en diagramas ANSI y se definieron los aprendizajes esperados según los módulos. Los componentes de cada módulo fueron lecciones de aprendizaje y evaluaciones sumativas de inicio cuya finalidad era estimar cuánto se sabía y cuánto se aprendió en las lecciones de aprendizaje. Se inscribieron 142 estudiantes voluntarios de licenciatura y posgrado; se obtuvieron los registros de la actividad realizada en el curso a través del Diagrama de informe e Informe completo que arroja el campus Ciencia CUVED, hecho en Moodle y que hospeda el curso. Bajo un diseño de series de tiempo descriptivo, se obtuvo las rutas de aprendizaje de los estudiantes participantes y se analizó su tendencia de forma visual y descriptiva. Los resultados mostraron que los elementos de evaluación sumativa iniciales y finales, como las lecciones de aprendizaje que componen los módulos del curso, permitieron a los estudiantes retroalimentar su aprendizaje y elegir las rutas de aprendizaje que deseaban seguir. Según los modelos encontrados a través del análisis de tendencia, las rutas seguidas por los estudiantes fueron de tipo no lineales, polinomiales de cuarto grado.

Palabras Clave: Curso autogestivo modular en línea, metodología de la investigación, aprendizajes esperados, universitarios, autorregulación, rutas de aprendizaje, Moodle.

\section{Abstract}

The objective of this work was to design a research methodology course, that will be self-managed, modular and online, aimed to university students; as well as to describe the students' learning pathway. In order to design this seven-module course, the e-course model based on experience was followed. In the process, a structure of the self-managed and modular course on ANSI diagrams was obtained; and the expected learning outcomes according to the modules were defined. The compo-

1 Directora del Laboratorio Digital de Desarrollo Infantil, Carrera de Psicología a Distancia, UNAM, México, esperanzagr@gmail.com 2 Coordinador de desarrollo y becarios del Laboratorio Digital de Desarrollo Infantil, UNAM, México, argenisespinoza25@gmail.com 3 Director del Laboratorio de Evaluación y Educación Digital, Carrera de Psicología, UNAM, México, arturomeister@gmail.com 4 Coordinador de proyectos del Laboratorio de Evaluación y Educación Digital, Carrera de Psicología, UNAM, México, jomss777@ gmail.com 
nents of each module were lesson plans and initial summative assessments whose objective was to estimate how much they knew and how much they learned in the course lessons. 142 undergraduate and graduate volunteer students were enrolled. The records of the activities carried out during the course were obtained through the Report Diagram and the Complete Report that the CUVED Science Campus provided, which is in moodle and hosts the course. Under a descriptive Time Series Design, the students' learning pathways were obtained and their tendency was analyzed in a visual and descriptive way. The results showed that the elements of the initial and final summative assessment, as well as the lessons that constitute the modules of the course, allowed the students to reflect on their own learning and to choose the learning pathways they wanted to follow. According to the models found through the trend analysis, the students followed a nonlinear, fourth-degree polynomial route.

Keywords: Self-managed modular online course, research methodology, expected learning outcome, university students, self-regulation, learning pathways, Moodle.

\section{INTRODUCCIÓN}

El método científico como parte de la ciencia es una forma de pensar y trabajar para lograr un conocimiento más completo del mundo prediciendo lo no observado y construyendo objetos funcionales (Rossiter 2006). En el ámbito educativo, el método científico es una competencia que debe enseñarse para que el alumno aprenda a hacer investigación. Su enseñanza comprende siete dimensiones: propuesta de investigación, diseño del estudio, planificación, recolección y procesamiento de datos, análisis de datos, conclusiones, y comunicación de resultados. Además de esto, es importante que los estudiantes adquieran una actitud crítica y desarrollen la capacidad de trabajar en equipo (Fossion y Zapata, 2015). En el ámbito universitario, su enseñanza se ha limitado a la exposición y presentación de conocimientos y procedimientos ya elaborados, dejando de lado estas siete dimensiones, ya que a los alumnos se les presenta conocimientos especializados relacionados a distintas metodologías prestando muy poca atención al establecimiento de estas competencias, a la aplicación del proceso de investigación científica y a las necesidades actuales de la sociedad.

Es común que la enseñanza del método científico en los estudios de pregrado adopte un modelo enciclopédico de tipo revisionista, impidiendo con esto el desarrollo en los alumnos de habilidades y competencias para investigar. Por consiguiente, el estudiante de licenciatura no tiene la oportunidad de conocer y reflexionar sobre las características que comprende el método científico (Gugliano y Robertt, 2010). Recientemente se han hecho intentos para dejar este tipo de enseñanza sustituyéndola por prácticas pedagógicas basadas en evidencias de aprendizaje en las que se recolecta información sobre los efectos de determinadas variables en el problema de interés y su relación con la forma de plantear las hipótesis (Johnson \& Gotthard, 2015).

De esto se desprende la necesidad de que se enseñe a los alumnos de pregrado la aplicación de la ciencia en ámbitos particulares y así impulsar en los estudiantes, mediante actividades de investigación, la capacidad de construir conocimiento científico, como también que use y maneje adecuadamente una metodología científica de investigación (Lourenco et al., 2014).

Considerando lo anterior, el objetivo de este artículo fue diseñar un curso autogestivo modular en línea para ensenar metodología de la investigación a universitarios. El curso permitiría describir la ruta de aprendizaje que presentan los universitarios en la adquisición de habilidades de investigación que les permitan reflexionar sobre el 
papel de la metodología científica en la creación de conocimiento y la aplicación de este en la solución de los problemas que enfrenta la sociedad. Se supuso que las rutas de aprendizaje serían trayectorias no lineales, para lo cual se usó análisis de tendencia ajustándolo a modelos matemáticos que expliquen las rutas seguidas por los estudiantes.

A diferencia de la enseñanza enciclopédica revisionista que recurre a la memorización de conceptos de metodología científica, el curso está diseñado para permitir que el estudiante resuelva problemas tomando decisiones sobre el análisis estadístico que puede usar en la investigación, además de navegar en el curso sin tener que ser guiado por un profesor o tutor. El estudiante puede seguir una sola ruta de aprendizaje, según sus necesidades o intereses. Bajo estos supuestos, la hipótesis de este trabajo supone que el curso autogestivo modular en línea diseñado para enseńar metodología de la investigación a universitarios permite describir las rutas de aprendizaje que presentan los universitarios. Estos, independientemente de cuál sea la ruta seguida, culminan satisfactoriamente el curso.

En este artículo, se presenta en primer lugar cómo se ha enseñado la metodología de la investigación en ambientes presenciales y algunas propuestas que ayudaron a mejorarlo. En seguida, se hace mención de la forma cómo fue enseñada la metodología de la investigación en ambientes virtuales. En el siguiente apartado se expone los fundamentos teóricos del enfoque pedagógico que guiaron el diseño del curso autogestivo modular en línea de metodología de la investigación para universitarios. Posteriormente, se presenta la metodología para la construcción del curso mediante la utilización de diagramas de flujo de la forma en que está estructurado modularmente y su funcionamiento. Por último, se describen los resultados obtenidos en la implementación de la versión beta del curso autogestivo modular en línea de metodología de la investigación dirigido a estudiantes universitarios, a través del análisis visual de la tendencia que muestran las rutas que los estudiantes seguían al estudiar de forma independiente sin profesores y en las situaciones y problemáticas que se les presentaron en la interfaz del curso.

\section{ENSEÑANZA DE LA METOdOlogía DE}

\section{LA INVESTIGACIÓN EN AMBIENTES}

\section{UNIVERSITARIOS}

A lo largo del tiempo, la enseñanza del método científico no ha sufrido grandes cambios en el aula. Por ejemplo, Rizo (2010) dice que solo se ha centrado en la interacción profesor-estudiante mediante el aspecto teórico-práctico, en respuesta a dicha problemática. Colina (2007) propone un nuevo paradigma en el que las instituciones de nivel superior deben enfocarse en crear planes y programas y efectuar cambios curriculares para que los estudiantes sean capaces de incrementar sus capacidades científicas, construyendo y resolviendo problemas sociales y naturales diversos. Una experiencia exitosa al hacer esto fue el trabajo de González y Rodríguez (2016), en el que el tiempo de exposición en clase fue sustituido por espacios de reflexión en donde los estudiantes de nivel superior, a partir de recursos didácticos que revisaban en su hogar, reflexionaban sobre cómo resolver los problemas revisándolos o analizándolos en sesiones de colaboración y discusión entre todos los estudiantes y el profesor de clase, lo que redituó en mejores resultados de aprendizaje de los estudiantes.

Como se mencionó anteriormente, debido a que las instituciones de educación superior deben ajustarse y dar respuesta y solución a las necesidades sociales actuales, Scribano, Gandia \& Mangallanes (2006) afirman que es de vital importancia reparar la brecha existente entre la enseñanza de la metodología de la investigación y la realidad social. Rizo (2010) menciona que esto se debe a que se siguen presentando problemas de organización y presentación del conocimiento. Estos problemas radican en que dicho conocimiento se aborda mediante procesos en cadena, sin tomar en cuenta que la formación en investigación sería más eficaz si el estudiante asumiera la investigación como algo en lo que está involucrado, ya que es un quehacer práctico vinculado con los problemas y necesidades sociales comprometidos. Por ejemplo, una manera de involucrar a los estudiantes con los problemas y necesidades sociales es ponerlos en contacto desde la infancia con dichas problemáticas, como se hizo en Alemania, donde 
a los niños de 8 a 12 años de edad les enseñaron a aprender el método científico en el contexto de la paleontología y el cambio climático (Leonard et al., 2016).

Pese que a nivel escolar la ciencia y su enseñanza son reducidas a la exposición y presentación de conocimientos y procedimientos ya elaborados, en la literatura revisada, se han hecho distintas propuestas para potenciar su enseñanza. Por ejemplo, Sánchez (2014), propone una dinámica de enseñanza de la metodología de la investigación basada en prácticas concretas. Dicha propuesta se resume en 4 puntos específicos vinculados entre sí: i) investigar teniendo como referente el mismo conocimiento científico, ya sea desde la enseñanza de procesos, mecanismos u operaciones llevadas a cabo en el ejercicio científico; ii) elaborar una didáctica de enseñanza práctica de las operaciones que se llevan a cabo en un proceso científico; iii) planear estratégicamente la didáctica de enseñanza en cada uno de los niveles o grados escolares y iv) contar con un espacio que tenga las herramientas apropiadas y estar aliado a otro investigador activo en un ambiente universitario, con el fin de que los estudiantes puedan conocer, reflexionar y comprender las características del método científico. Agregado a esto, Lourenço, Costa y Hernandes (2009) llevaron a cabo dicho proceso en un ambiente universitario y se dieron cuenta que ayuda a que los estudiantes tengan un panorama más amplio de su campo y actividad profesional.

\section{ENSEÑANZA DE LA METOdología dE}

\section{LA INVESTIGACIÓN EN AMBIENTES}

\section{VIRTUALES}

Dado que hay sectores donde los ambientes virtuales no son contemplados como un medio efectivo y de calidad para la enseñanza, la percepción que se tiene sobre la calidad y efectividad de los entornos virtuales, como lo demostró un estudio de Walter (2016), varían entre aquellas que consideran que son entornos unidireccionales de comunicación y que solo sirven para proporcionar a los estudiantes materiales adicionales de la clase presencial. También que solo son complementos de las clases curriculares de las instituciones, las mismas que son una manera distante de establecer relaciones de aprendizaje entre estudiantes y profesores. Por último, son una oportunidad de comunicación que permite desarrollar una enseñanza con aprendizajes significativos para los estudiantes.

En la actualidad, las instituciones de educación enfrentan el reto de incorporar procesos educativos no presenciales soportados por la tecnología, en los que se logre una comunicación efectiva, no solamente en términos de transmisión de la información, sino también de conocimientos, habilidades y actitudes para el aprendizaje (Usoro \& Akuchie, 2012). En respuesta a dicho reto, en la actualidad existen diversos cursos de metodología de la investigación en ambientes virtuales, los cuales constan de variadas modalidades de interacción y elementos multimedia que respaldan o apoyan la adquisición del conocimiento. En términos de la enseńanza de metodología de la investigación, existen distintos esfuerzos realizados por universidades, tales como el de Atlantic International University, la Universidad de Navarra, la Universidad de Guadalajara y sitios en línea de acceso libre como Pupilum con un curso de investigación clínica de Rodríguez y Martín (2016).

En primera instancia, el curso de metodología de la investigación ofrecido por la Atlantic International University, tiene como objetivo general lograr que el estudiante sea capaz de analizar las bases teórico-metodológicas de la investigación. Brinda al estudiante 10 lecciones de contenido complementadas por distintos recursos, tales como: lecciones en formato de audio, videoconferencias, materiales de lectura y exámenes. Los temas que abarca son: la investigación, elementos del proceso de investigación, los temas de la introducción y marco teórico de la investigación, inteligencia, hipótesis de investigación, técnicas de muestreo, cálculo de tamaño muestral, recolección y procesamiento de datos, redacción del proyecto de investigación y metodología a utilizar.

Por otra parte, la Universidad de Navarra, dentro de los cursos de posgrado en el área de medicina, ofrece un curso de metodología de la investigación en el que se cubren diversos temas, tales 
como: conceptos básicos de la investigación, bases de datos, inicio del análisis estadístico, estadística aplicada básica, introducción al SPSS, ética de la investigación biomédica, estadística multivariante e interpretación de resultados.

A diferencia de los anteriores, el curso de metodología de la investigación del sitio de acceso libre Pupilum está enfocado en el área clínica. Además de no estar sujeto a un calendario fijo, es decir que puede ser cursado al ritmo preferido por el usuario, también ofrece materiales de estudio, como archivos PDF, certificado oficializado y una gran cantidad de profesores con el fin de brindar ayuda o tutoría a los alumnos. Los temas retomados en este curso son; 1) Método Científico, 2) ¿Cómo se generan preguntas en investigación clínica?, 3) Búsquedas bibliográficas, 4) La lectura crítica de artículos científicos, 5) Objetivos e Hipótesis, 6) Diseño y tipos de estudios, 7) El análisis de datos, 8) La presentación de resultados y 9) Cómo diseñar un proyecto de investigación (Rodríguez y Martín, 2016).

Otras universidades y sitios en línea se han dado a la tarea de ofrecer cursos sobre metodología de la investigación y manejo del paquete estadístico SPSS. Entre algunas de ellas se encuentran: La Universidad de Guadalajara con la Red Universitaria de Jalisco, donde ofrecen un curso de metodología de investigación. El objetivo de este es que el participante desarrolle la capacidad de diseñar proyectos de investigación científica cubriendo cuatro ejes centrales en su contenido: análisis de la realidad, naturaleza y límites de la investigación y manejo del marco teórico, así como conceptos clave. Por otra parte ofrece un curso de capacitación y manejos de SPSS, cuyo objetivo es que el estudiante comprenda la diferencia entre investigación cualitativa y cuantitativa, enseñar los métodos y metodologías que se han asumido en cada una de ellas, y enseñar a utilizar dos de las principales herramientas informáticas en la investigación (EXCEL y SPSS). Sin embargo, aún no se unifica la enseñanza del método científico con el manejo de distintos paquetes de análisis estadístico en cuanto a cursos en línea.

Estos esfuerzos responden a las nuevas tendencias de la nueva era de la sociedad del conocimiento y la interacción, en la cual las tecnologías de la información y la comunicación tienen un papel determinante. Es por esto que es de suma importancia considerar el uso de dichas tecnologías en los distintos niveles educativos, así como desarrollar un enfoque pedagógico sólido que busque generar en los estudiantes las competencias necesarias para la aplicación de la metodología de la investigación en una serie de cursos que se desarrollarán y que se explican en este artículo. El primer curso de esa serie, que busca específicamente que el estudiante pueda implementar técnicas y programas para el análisis de resultados desde el método científico, cubriendo así las necesidades de la sociedad en problemas reales. En este sentido, en el curso estudiado en la presente investigación se unificarán las bondades de los ambientes presenciales y virtuales; además, se fortalecerán las debilidades que estos han tenido a lo largo del tiempo hasta llegar a la promoción del autoaprendizaje en situaciones donde los estudiantes reflexionan, usan y aplican el análisis de resultados.

\section{FundAMENTOS TEÓRICOS DEL ENFOQUE PEDAGÓGICO PARA EL DISEÑO DEL CURSO AUTOGESTIVO MODULAR EN LÍNEA.}

De acuerdo al objetivo planteado, el diseńo del curso autogestivo modular en línea de metodología de la investigación para universitarios se fundamenta en The Learning Outcomes (Ma Watson et al., 2016; Simmons, 2013) o resultados de aprendizaje/aprendizajes esperados, también se fundamenta en la creación de entornos tipo metatutor de Taub, Azevedo, Bouchet $\&$ Khosravifar (2014), donde el estudiante implementa estrategias de aprendizaje autorregulado, como lo indica Azevedo et al. (2009), ya que los estudiantes son quienes gestionan su proceso de aprendizaje. En el referido curso se utilizan estrategias cognitivas, motivacionales, metacognitivas y de apoyo para construir su conocimiento de forma significativa (Núñez et al., 2006). El ambiente de aprendizaje autogestivo es posible ya que el curso fue diseñado de tal forma que los recursos, actividades, evaluaciones con una estructura autodirigida de ruta libre de navegación permite estudiar de forma au- 
togestiva y dirige el aprendizaje del estudiante de la misma forma.

Aguilera (2005) indicó que la educación debe lograr ser autónoma para conducir su proceso de aprendizaje, con las técnicas, herramientas y contenidos necesarios, sin depender necesariamente de la figura del maestro. A este proceso, Aguilera le llama autogestión, que es el fin del curso que se presenta en este artículo. El curso fue creado pensando en crear un ambiente de aprendizaje autogestivo, que lleve al estudiante a gestionar sus procesos de pensamiento y aplique conocimientos para aprender la metodología y aplicarla en sus investigaciones.

Asimismo, Ponce (2016) plantea la respuesta a preguntarse ¿qué hace que un entorno virtual de aprendizaje sea autogestivo? Ella dice que a un entorno virtual lo hace autogestivo todo aquello que fortalezca la capacidad del estudiante para tomar decisiones sobre su propio aprendizaje y le permita administrarlas siendo responsable de sí mismo. Además ese ambiente debe ser lo suficientemente interactivo con calidad y eficiente bidireccionalidad para que el estudiante deje de ser observador para y se convierta en un participante activo que emita, reciba e interprete información.

Además, las características de un curso modular donde el estudiante elige los módulos que considera pertinentes necesitan estudiar y tomar el orden que desea para estudiar, así como recibir retroalimentación de sus acciones. Esto permite que el estudiante lleve un proceso de aprendizaje autogestivo en el que se puede dar también la autorregulación.

La autorregulación y el aprendizaje autogestivo constituye un ámbito de creciente interés por la manera en que estos aprendizajes influyen en la forma de comprender los problemas y desenvolverse en entornos virtuales. Esta estrategia tiene implicaciones importantes en el diseño de dichos entornos, ya que es posible diseñarlos adaptándolos a las necesidades de aprendizaje de los propios estudiantes (Taub et al., 2014). En este sentido, los alumnos realizan el curso a su propio ritmo, definiendo también su propia ruta de aprendizaje.
Dicha ruta está en función de sus propias necesidades e intereses, según los aprendizajes esperados, enunciados de manera que denoten acciones directamente relacionadas a la competencia que se busca generar en cada módulo, como lo recomiendan Ponce (2016) y Ma Watson et al. (2016). Esto está apoyado por diversos recursos multimedia, tales como: videos, presentaciones de PowerPoint, archivos de audio, entre otros; además cuenta con lecciones de contenido que constan de textos, gráficos, videos, imágenes, audios e interactividad en forma de problemas formulados como preguntas y comentarios con retroalimentación inmediata, donde el contenido está dosificado para una mejor comprensión.

Por último, tal como lo recomiendan Azevedo et al. (2009), el ambiente virtual de aprendizaje autogestivo modular del curso es un ambiente de aprendizaje hipermedia diseñado para permitir a los estudiantes generar y comprobar hipótesis insertándolos en un contexto de aprendizaje particular en el que los participantes apoyarán su aprendizaje, ya que: 1) les permite tomar decisiones de instrucción con respecto a sus metas y objetivos de aprendizaje; 2) se inserta en un contexto de aprendizaje particular donde los estudiantes deben elegir los medios para facilitar su aprendizaje; 3) la estructura de los cursos autogestivos modulares en línea sugieren a los estudiantes los procesos de regulación autónomos, cognitivos y de motivación; 4) En algunos casos, es un contexto donde agentes artificiales pueden apoyar el aprendizaje de los estudiantes y fungir como agentes reguladores y 5) es inculcada la autorregulación, antes durante y después del curso.

\section{Materiales y Métodos}

\section{Participantes}

En trabajos de este tipo de investigación e innovación tecnológica educativa con participantes se debe considerar dónde se presenta el diseño e implementación de una innovación educativa al equipo desarrollador y los participantes como usuarios finales de los desarrollos que se ponen a su disposición. Bajo el modelo metodológico que guio las acciones de este estudio se requirieron 
catorce expertos: siete diseñadores de contenido y siete diseñadores instruccionales, más los administradores y asesores de soporte.

El tipo de muestro fue no probabilístico, pues una vez que el equipo diseñador concluyó el curso autogestivo modular en línea de metodología de la investigación para universitarios, se abrió la convocatoria mediante Internet, por redes sociales de la comunidad universitaria, principalmente de la Universidad Nacional Autónoma de México para cursar la versión Beta 1.0. Solo se especificó que el curso era para estudiantes de licenciatura o de posgrado. Se inscribieron 142 participantes de manera voluntaria, quienes manifestaron su interés por vivir la experiencia de un curso de esta naturaleza; 102 eran mujeres y 40 hombres. El rango de edad fue de 19 a 56 años, con una media de 34 años. El 66.03\% eran estudiantes de licenciatura y el resto estudiantes de posgrado. El $89 \%$, de psicología; el 6\%, de biología; el 4\%, docentes y el $2 \%$, de sociología. El $70 \%$ contaban con acceso a Internet desde casa; el 20\%, desde el trabajo; el $8 \%$, desde la universidad y el $1 \%$, desde lugares públicos.

\section{Recolección de datos}

En el curso autogestivo modular en línea de metodología de la investigación se recolectaron los datos que arroja el campus virtual Ciencia CUVED, donde se hospeda el curso a través del Diagrama de informe e Informe completo (Arratia, Galisteo, Pérez y García, 2009), que contiene la información de la labor desarrollada por los estudiantes mediante un listado completo de las actividades del curso con indicación del número de acciones realizadas en cada una de ellas y la fecha de la última acción. Asimismo, se obtiene la descripción detallada de cada actividad realizada desde el inicio hasta el final del curso y la distribución de registros agrupados por día. Con estos informes se pudo describir la ruta de aprendizaje que seguían los estudiantes, entendida como las acciones que decidían realizar según la estructura modular que les ofrecía el curso.

\section{Diseño}

Para describir las rutas de aprendizaje de los estudiantes, se tomó un diseño de seguimiento como base de este estudio. El diseño de series temporales descriptivas o diseños funcionales (Silva, 2004) permitió este fin, porque presenta los datos sin implicación de alguna intervención, más que los registros en los informes que arroja el campus virtual Ciencia CUVED, que hospeda el curso, para mostrar la evolución de los datos a lo largo del tiempo durante el cual estuvo disponible, es decir: cuarenta y siete días.

\section{Modelo de diseño del curso}

El modelo de diseño del curso autogestivo modular en línea de metodología de la investigación que se adoptó fue The Experience-based e-course design model (Tüzün \& Çınar, 2016) o modelo de diseño de e-curso basado en la experiencia, que consistió en seis etapas: formar el equipo de diseño y búsqueda preliminar de contenido e insumos, el análisis por matrices de competencias y aprendizajes esperados, análisis de la instrucción, el diseño técnico, la integración del desarrollo, pruebas y mejoras. Este modelo de diseño es una metodología completa que permite el mantenimiento de los esfuerzos de construcción de los cursos para Web pensados en ciclos integrados que permiten la implementación del proceso de diseño de manera más eficaz y, consecuentemente, obtener mayor calidad en el producto final.

\section{Procedimiento}

De acuerdo al modelo de diseño de e-curso basado en la experiencia para desarrollar el curso e implementarlo y poder describir las rutas de aprendizaje de los universitarios se realizaron las siguientes etapas.

Como se expuso en la introducción de este artículo, se han hecho innovaciones tecnológicas para mejorar la enseñanza de la metodología de la investigación en diferentes universidades. Para ello, los cursos web requieren de innumerables horas de trabajo, de personas especialmente capacitadas para cumplir con dicha labor. Este curso no fue la excepción. Por esto se siguieron una serie de 
etapas que se describen a continuación:

i. En esta etapa se formó el equipo y se buscaron los insumos para el desarrollo del curso. Se realizó la formación y capacitación de un equipo de profesionales y alumnos con experiencia en diseño de la instrucción y contenido. Se les capacitó en edición multimedia, de imágenes en Photoshop, video con Premiere, creación de Slideshows con Cincopa, creación de escenas animadas en video con Goanimate y diseño de juegos interactivos con Educaplay. No se enseñó el uso de las herramientas de forma general; se formó al equipo en los estándares que el curso autogestivo modular en línea debía tener en imágenes, banners, botones, slider, juegos interactivos y videos, los cuáles son insumos necesarios para el diseño del contenido y actividades de los módulos que componen el curso. También se les capacitó en principios básicos de lenguaje HTML y jQuery para poder hacer la composición interactiva del curso. Otro aspecto muy importante en la capacitación fue en el uso y gestión de Moodle para crear los espacios virtuales del curso. La interfaz de forma modular fue posible gracias a la versatilidad de Moodle en el campus Ciencia CUVED. ii. Después de la capacitación, en la segunda etapa se hizo el análisis por matrices de competencias y aprendizajes esperados.

iii. En la tercera etapa se hizo análisis de la instrucción que consistió en generar los contenidos de metodología de la investigación y estadística.

iv. En la cuarta etapa se realizó el desarrollo pedagógico del curso en guiones técnico-pedagógicos de contenido y matrices de aprendizajes esperados para cada módulo del curso.

v. Después de ello, en la quinta etapa se hizo el hospedaje en los servidores y la aplicación de construcción técnico-pedagógica, donde se creó el curso, las evaluaciones formativas y sumativas de los aprendizajes, ejercicios interactivos autoaplicables (g-learning), actividades prácticas simuladas, emisión de constancias y créditos académicos, obtención de informes y seguimiento del participante, administración del curso, gestión de usuarios, monitoreo permanente 24 x 7 × 365 (servidor y plataforma) $\mathrm{y}$ respaldos del curso.

Ahora bien, con respecto al contenido del curso, se crearon siete módulos: medición en la ciencia, variables, escalas de medición, descripción y orga-

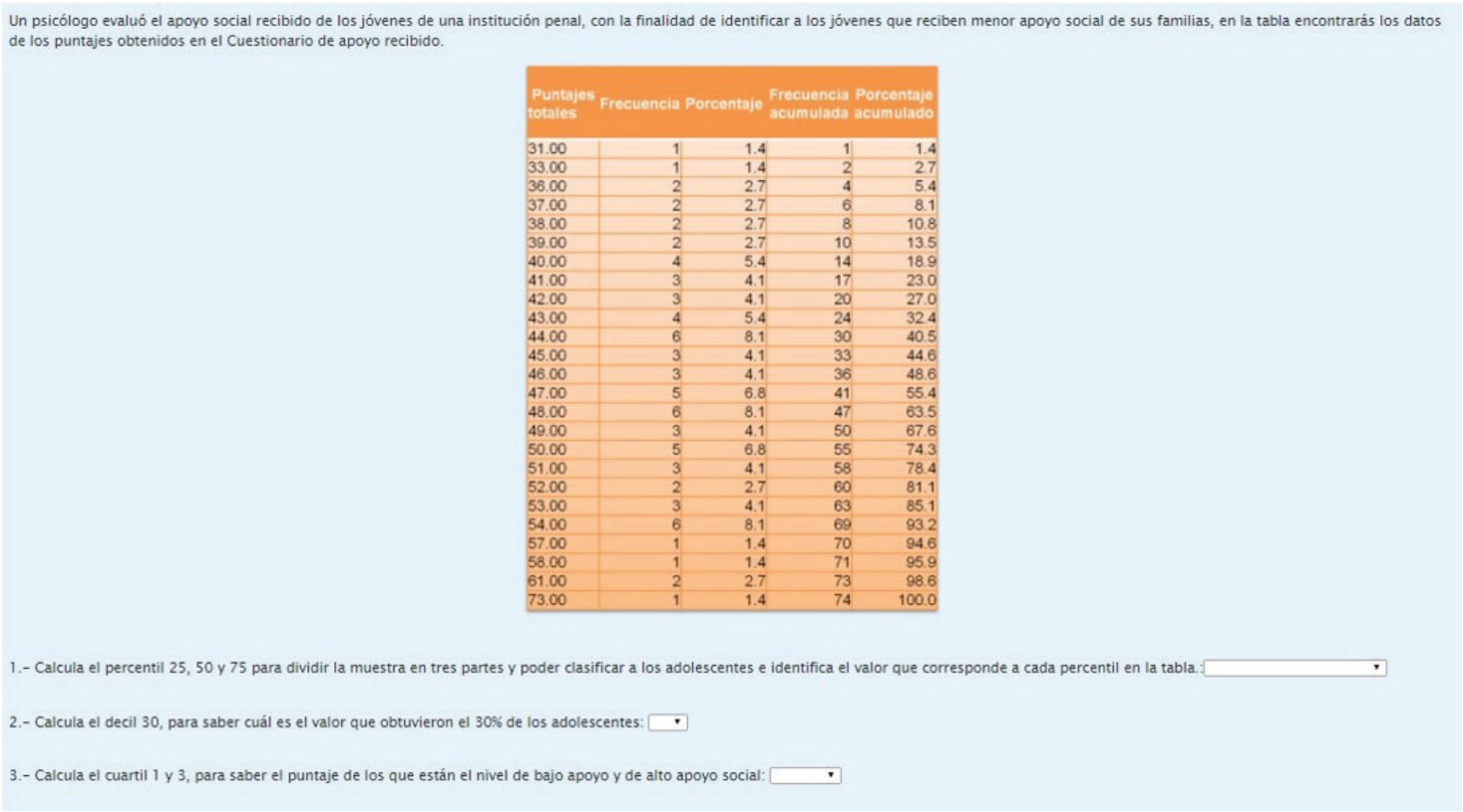

Figura 1. Ejemplo de ejercicio interactivo autoaplicable 
nización de datos, medidas de tendencia central, medidas de dispersión y tablas y figuras. Cada uno articula sus contenidos por competencias y aprendizajes esperados. El formato de presentación es en lecciones con una navegación lineal, para lo que se presentan conceptos y problemas a resolver con retroalimentación inmediata y automática. En las páginas de la lección se intercalan los ejercicios autoaplicables que simulan problemas con un carácter investigativo (ver figura 1).

Estos ejercicios autoaplicables son escenarios de simulación de casos de investigación que ayudan a crear experiencias de aprendizaje auténticas, permitiendo con esto (como lo recomienda Guinne, 2012) que los entornos virtuales promuevan la transferencia de las habilidades aprendidas en espacios de simulación. El entorno virtual del curso autogestivo modular en línea de metodología de la investigación está diseñado para estimular la motivación en los estudiantes mediante retroalimentaciones con las que se busca fortalecer sus aprendizajes (Duffy y Azevedo, 2015).

Los ejercicios autoaplicables se presentan como juegos tipo g-learning, videos, imágenes y bibliografía recomendada para apoyar o complementar el conocimiento adquirido. Esto hace más rico el entorno virtual y genera experiencias de aprendizaje más significativas superando el efecto convencional de, por ejemplo, las conferencias presenciales con resultados de aprendizaje menos significativos que un entorno virtual diseñado adecuadamente, donde el estudiante interactúa con las situaciones creadas para que tome decisiones aplicando el conocimiento (Park et al., 2014).

Por otra parte, debido a que la interacción entre participantes puede apoyar el aprendizaje en los estudiantes, en el curso autogestivo modular se cuenta con un muro de "comentarios", donde aportan ideas con la finalidad de fomentar una conversación general de los temas tratados (Sander \& Golas, 2013).

Una vez concluido el diseño del curso, según el modelo e-curso basado en la experiencia, como sexta etapa y final siguieron las pruebas y mejoras. Para ello se abrió la convocatoria a través de redes sociales para cursar la versión Beta 1.0. Los estudiantes que cursaron emitieron su opinión y se hicieron las mejoras en el diseño gráfico, en su mayoría; y en menor proporción, en la configuración de ciertas secuencias de algunos ejercicios autoaplicables que debieron modificarse para mejor navegación en el curso.

\section{Funcionamiento Del CURSO}

\section{AUTOGESTIVO MODULAR EN LÍNEA}

El curso autogestivo modular en línea de metodología de la investigación está dirigido a establecer competencias relacionadas con los conocimientos declarativos, demostrativos y también a establecer competencias procedimentales que doten al estudiante de las habilidades para utilizar la metodología y estadística en la investigación de los fenómenos de las ciencias sociales y de la salud. Para ello se buscó una estructura modular y flexible idónea que permita establecer dichas competencias, donde el estudiante elige la ruta que desea seguir, tomando los módulos en el orden que decida según sus necesidades y evaluando los conocimientos que posee para determinar qué debe estudiar para desarrollar las habilidades en metodología de la investigación deseadas, considerando la culminación del curso como una meta lograda.

La estructura del curso se presenta en diagramas de flujo tipo ANSI ${ }^{5}$ (figura 2). El curso autogestivo modular en línea consta de tres momentos determinantes: ¿Cuánto sabes?, que es la evaluación que permite al estudiante valorar sus conocimientos actuales; "Aprende con la lección", que es la lección de evaluación formativa con la que se estudia y se resuelven problemas en ejercicios interactivos autoaplicables para aprender habilidades de metodología de la investigación que le hacen falta y le fueron indicadas en la evaluación sumativa que se realizó primero en ¿Cuánto sabes?; por último: ¿Cuánto aprendiste?, que es la evaluación sumativa que se realiza cuando ya se ha alcanzado el aprendizaje deseado al resolver los problemas planteados en las varias veces que se estudió en "Aprende con la lección” (ver figura 2).

El curso autogestivo modular en línea, consta de tres momentos determinantes ¿Cuánto sabes? Es la evaluación que permite al estudiante valorar sus 


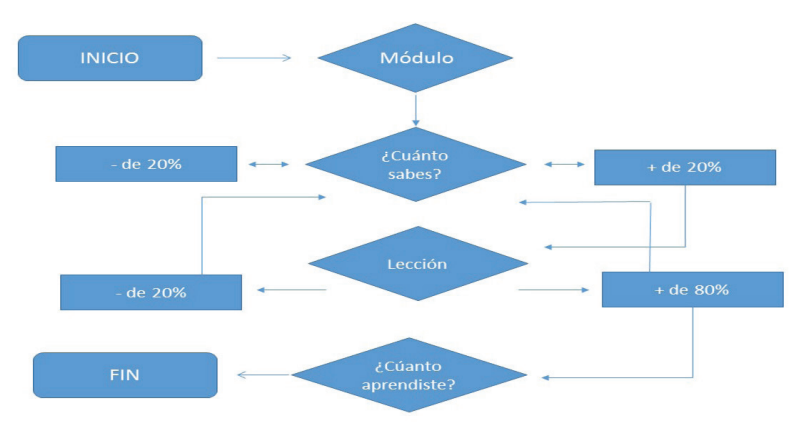

Figura 2. Diagrama de flujo de un módulo

conocimientos actuales; "Aprende con la lección" Es la lección de evaluación formativa con la que se estudia y se resuelven problemas en ejercicios interactivos autoaplicables para aprender habilidades de metodología de la investigación que le hacen falta y le fueron indicadas en la evaluación sumativa que se realizó primero en ¿Cuánto sabes?; por último ¿Cuánto aprendiste? Es la evaluación sumativa que se realiza cuando ya se ha alcanzado el aprendizaje deseado al resolver los problemas planteados en varias veces que se estudió en "Aprende con la lección" (Ver Figura 2).

Antes de iniciar el curso, el primer módulo es la lección "para comenzar", donde se indica al estudiante el funcionamiento general de la interfaz, los medios y criterios para cursar, estudiar, evaluarse, retroalimentarse, lo que le otorga al estudiante la posibilidad de planificar sus acciones y decidir qué ruta seguir o cómo organizar su tiempo de dedicación a un determinado módulo. Es una lección que permite establecer la autorregulación básica. Una vez terminada, el estudiante podrá ingresar libremente al módulo que sea de su elección, guiándose con algunos criterios que se le dan a conocer en ¡Para comenzar! Primero debe valorar con la evaluación sumativa “¿Cuánto sabe?”, qué conocimiento tiene actualmente. Si obtiene una calificación menor al $20 \%$ se le recomienda estudiar la lección "Aprende con la lección”, como evaluación formativa. Si obtiene un desempeño mayor al $80 \%$, puede realizar la evaluación sumativa denominada “¿Cuánto aprendiste?" De la misma manera, cuando ha estudiado con la lección y alcanza en ella un desempeño del $80 \%$, puede hacer la evaluación “¿Cuánto aprendiste?” (ver figura 3).

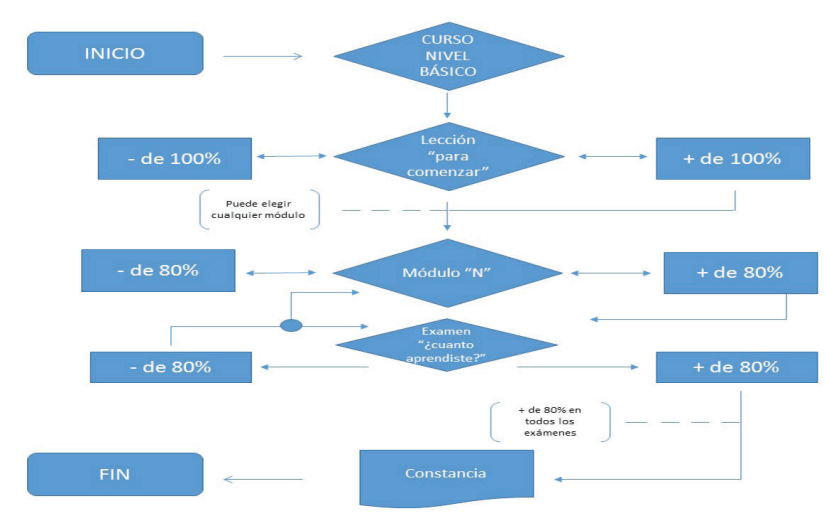

Figura 3. Diagrama de flujo del curso de la estructura modular del curso autogestivo modular en línea.

Una vez haya obtenido una calificación aprobatoria en todos los módulos, el estudiante podrá imprimir su constancia oficial del curso emitida por el proyecto que financió el diseńo del curso y de la UNAM.

Por otra parte, la lección de cada módulo está constituida por diversas actividades y recursos. Se presenta la información específica de cada módulo. Con respecto a los g-learning, estos son una herramienta cognitiva diseñada con el fin de reafirmar y retroalimentar el conocimiento, intercalado en el contenido. Los ejercicios autoaplicables dentro de la lección de contenido evalúan la comprensión del contenido teórico mediante problemas y el manejo de conceptos. Por último, se cuenta con una sección denominada "aspectos para recordar”, que sintetiza los conceptos clave de cada tema, así como también se recomienda bibliografía relacionada. El orden en el cual se presentan dichas actividades y recursos se muestra en la figura 4.

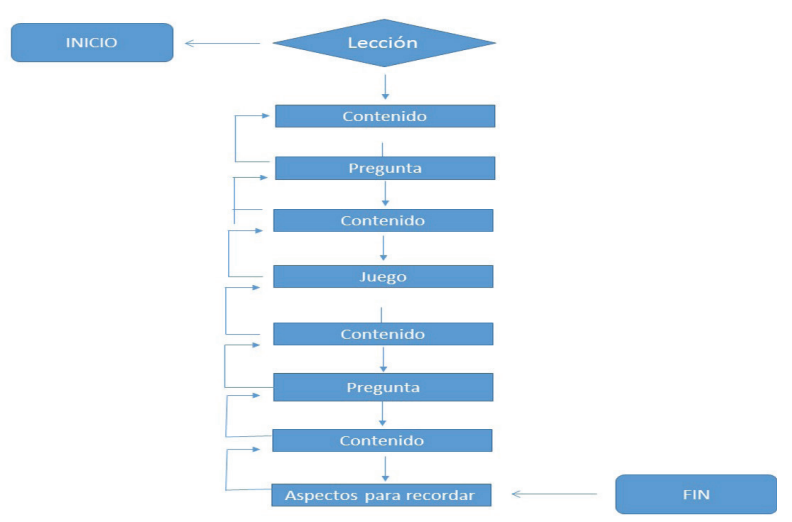

Figura 4. Diagrama de la estructura de una lección. 
Se observa que la estructura del curso autogestivo modular en línea muestra una configuración programada de tal forma que el alumno cuenta con la guía, su ruta de navegación. La secuencia que sigue depende de los conocimientos y habilidades que posee, los que aprende al resolver los ejercicios interactivos autoaplicables. Según el grado que logra mostrar en los aprendizajes que se espera obtenga, tiene opciones para repetir evaluaciones o lecciones por varias veces, según estime necesario, obteniendo un record acumulativo.

\section{Resultados}

Como se citó anteriormente, la presentación de los resultados se basó en la recolección de datos a través del Diagrama de informe e Informe completo (Arratia et al., 2009). Los datos procesados se representaron en gráficos lineales que muestran en el eje de las abscisas los días que estuvo disponible el curso, en total cuarenta y siete días, cuarenta días del tiempo regular y siete días de recuperación para quienes tuvieron problemas de ingreso al inicio con cuestiones de claves de acceso. En el eje de las ordenadas se muestra el número de acciones realizadas por los estudiantes, las cuales dan cuenta de la actividad interactiva. Se realizó un análisis de tendencia por análisis visual (Silva, 2004) para describir la ruta de aprendizaje de los estudiantes. Se puede ver las fluctuaciones, variaciones, aumentos y decrementos en los trayectos de actividad de los estudiantes. Se representan con modelos matemáticos que expresan la función bajo la cual se dan las rutas que siguen los alumnos. Graficando la línea de tendencia de estas rutas se sabe cuál es la mejor manera de representar la tendencia por el coeficiente de determinación que se asocia a cada modelo matemático. Si este índice está más cerca de 1 , se elige como mejor línea de tendencia. Se modelaron tendencias lineales, logarítmica y ponitómicas.

En principio, de los 142 estudiantes que se inscribieron voluntariamente, el 66\% acreditó el curso, es decir, fi- nalizaron todas las actividades de forma aprobatoria, lo que es un buen resultado considerando que el curso se dictó en diciembre durante el periodo vacacional y de fiestas navideñas.

Los informes generales mostraron por Moodle ciencia CUVED respecto a las visitas de las actividades independientemente del módulo de la lección. Tuvo 2,683 visitas; una cantidad mayor a cualquier otra actividad dentro del curso. Como se mencionó en secciones anteriores, en dicha lección se presenta una guía rápida y sintetizada de la dinámica de los aprendizajes que se espera obtengan los estudiantes bajo la estructura modular. También describe las actividades, evaluaciones e interfaz del curso. Esto muestra la recurrencia de los estudiantes a revisar y confirmar lo que pueden hacer, cómo hacerlo y lograr sus metas en el curso, como recordar las reglas y autorregular su actividad reafirmando las condiciones del curso. Tal vez su revisión constante muestra que no tenían un plan de acción de inicio y regresaban a recordar qué y cómo se puede navegar y alcanzar la meta de culminar el curso.

Respecto a la tendencia que mejor representó las trayectorias de las rutas que seguían los estudiantes se descubrieron formas polinómicas, lo que significa que las trayectorias tuvieron incrementos y decrementos relevantes en más de dos ocasiones. Esto se sabe porque los modelos matemáticos, es decir las ecuaciones que se obtuvieron, fueron ecuaciones de tercer a quinto grado.

Tabla 1. Modelos matemáticos obtenidos que describen las líneas de tendencias de las trayectorias que siguieron las rutas de aprendizaje de los estudiantes

\begin{tabular}{lll}
\hline \multicolumn{1}{c}{ Módulo } & \multicolumn{1}{c}{ Ecuación polinómica } & \multicolumn{1}{c}{$\mathbf{R}^{2}$} \\
\hline Actividades generales & $y=-0.0099 \times 3+0.8283 \times 2-22.456 x+235.18$ & 0.50821 \\
\hline $\begin{array}{l}\text { Módulo Medición en la } \\
\text { ciencia }\end{array}$ & $y=-2 E-05 \times 3-0.0933 \times 2+0.4272 x+165.74$ & 0.27074 \\
\hline Módulo Variables & $y=0.0082 \times 3-0.8145 \times 2+20.332 x-16.724$ & 0.34596 \\
\hline $\begin{array}{l}\text { Módulo Escalas de } \\
\text { medición }\end{array}$ & $y=0.0006 \times 4-0.0616 \times 3+1.8178 \times 2-11.167 x$ & 0.39582 \\
\hline Módulo Descripción y & $y=0.001 \times 4-0.1228 \times 3+4.3602 \times 2-40.538 x+$ & 0.48363 \\
organización de datos & 104.63 & \\
\hline Módulo Medidas de & $y=0.0008 \times 4-0.0861 \times 3+2.8043 \times 2-26.93 x+$ & 0.50766 \\
tendencia central & 56.652 & \\
\hline Módulo Dispersión & $y=7 \mathrm{E}-05 \times 5-0.008 \times 4+0.3361 \times 3-5.6488 \times 2+$ & 0.52641 \\
\hline Módulo Tablas y figuras & $y=-0.0009 \times 4+0.0522 \times 3-0.6358 \times 2-0.3033 x$ & 0.26094 \\
& +15.521 & \\
\hline
\end{tabular}


Como puede observarse en la figura 5, la lección “ipara comenzar!” tuvo un mayor índice de visitas en los primeros nueve días, lo cual denota que funcionó como una guía de inicio importante. En comparación se ven otros recursos dispuestos en el curso como una encuesta de opinión y la sección de obtención de la constancia, que fueron visitadas durante las últimas dos semanas con una frecuencia menor que la lección ¡Para comenzar!, incluso después de los veintinueve días de iniciado el curso. Ahora se presentan los resultados de las rutas de aprendizaje obtenidas por los estudiantes en cada módulo.

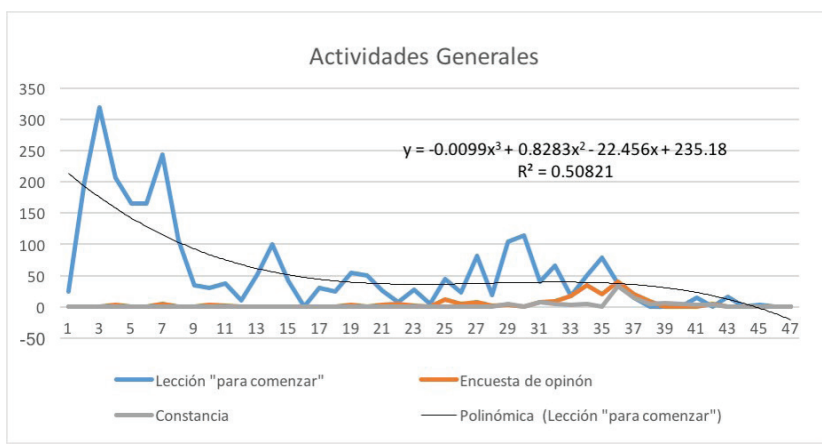

Figura 5. Visitas a las actividades de manera global.

La figura 6 muestra los registros de actividad en los tres componentes del módulo "Medición en la ciencia": ¿Cuánto sabes?, Aprende con la lección y ¿Cuánto aprendiste? La evaluación inicial de ¿Cuánto sabes? registró una mayor frecuencia de registros en los primeros diez días y después del día treinta y seis fueron casi nulos; Las visitas registradas en "Aprende con la lección" muestran una frecuencia variable con cierta constancia alcanzando un máximo de 560 registros el día siete. Por último, los registros de actividad de ¿Cuánto aprendiste? estuvieron por debajo hacia los cuarenta y siete días que estuvo abierto el curso. Sin embargo, tuvo menos intentos realizados porque aprendieron lo estudiado en la lección. Este módulo fue visitado con una mayor frecuencia en los primeros días del curso, pues al ser un curso autogestivo modular los participantes tenían la libertad de establecer sus rutas de aprendizaje a través de los distintos módulos ofrecidos. Se observó que fue elegido primero por los estudiantes, como si utilizaran la lógica lineal que se sigue en los cursos presenciales o en línea, así que ellos prefirieron empezar por comprender la concepción de la medición y su papel en la ciencia

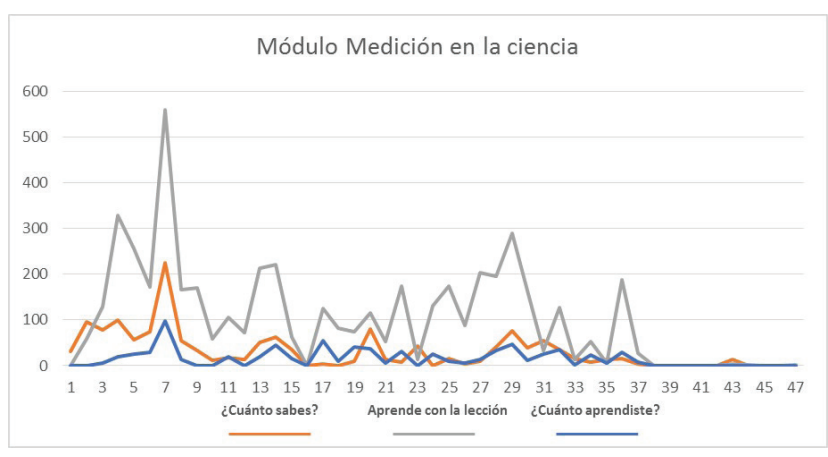

Figura 6. Actividad registrada en el módulo Medición en la ciencia.

En la figura 7 se distingue la variabilidad y afluencia de las evaluaciones sumativas inicial y final ¿Cuánto sabes? y ¿Cuánto aprendiste? En ambas fue similar en el módulo de variables; este módulo es el segundo del curso, pero el primero que aborda problemas aplicados sobre las variables en la investigación y su función metodológica. Se puede observar que "Aprende con la lección" fue el recurso más revisado por los estudiantes quintuplicando su actividad, con un intervalo de 50 a 250 registros diarios, en comparación con las evaluaciones sumativas del módulo. Probablemente aumentaba cada fin de semana, por los picos que se observan aproximadamente cada siete días. A diferencia de otros módulos, los estudiantes cursaron y finalizaron este de forma variada a lo largo de los primeros 37 días del tiempo disponible en el curso, lo cual respalda lo confirmado en estudios anteriores: los estudiantes permanecen en los cursos flexibles en tiempo, disponibilidad y accesibilidad (Rivera, 2011).

Aunado a esto, en la figura 8 se muestra una gran variabilidad con respecto al registro de las visitas en el módulo "Escalas de Medición" con una ligera carga a partir del día veinte al día treinta y ocho, ya que la frecuencia máxima fue de 317 registros en "Aprende con la lección", lo cual superó el módulo de variables. Con respectos a los registros de actividad en las evaluaciones sumativas ¿Cuánto sabes? y ¿Cuánto aprendizaje? se observa por debajo de la lección. Esto da cuenta 


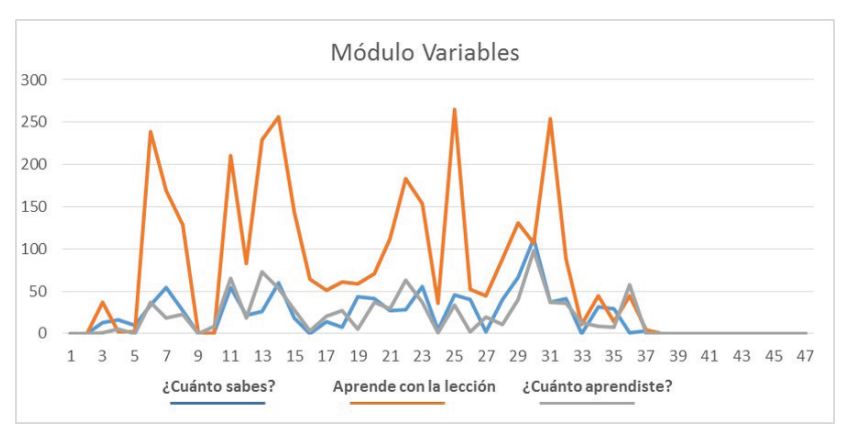

Figura 7. Registrado de la actividad del módulo variables.

de que los registros de interactividad en cada una de las actividades que pueden estar determinadas por la naturaleza de las misma (Peñalosa, 2010), como es el caso de la lección donde se presentan ejercicios autoaplicables interactivos, que demandan mayor interacción del estudiante. Además se muestra que desde el inicio del curso los estudiantes se interesaron por los contenidos de escalas de medición, ya que tuvo registros altos, aunque se incrementan hacia la cuarta semana, lo que indica que aún hay varios estudiantes que pudieron seguir una secuencia lineal de los módulos.

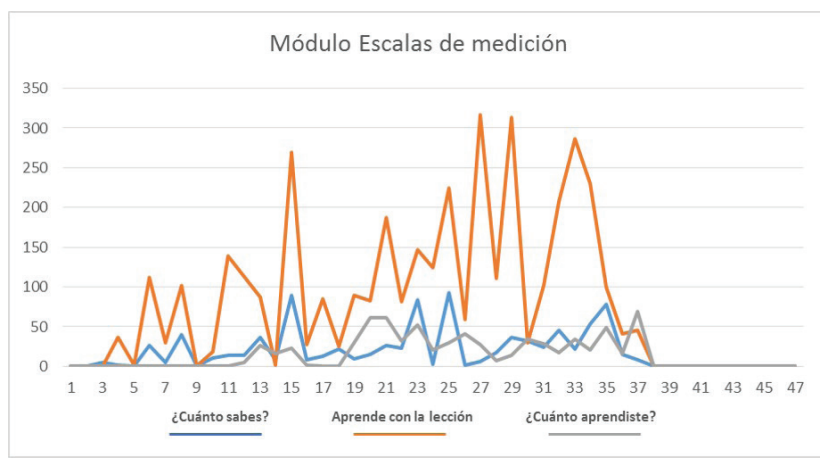

Figura 8. Registrado de la actividad en el módulo Escalas de medición.

Los registros de la actividad estudiantil en el módulo "Descripción y Organización de Datos" (ver figura 9) muestran un incremento considerable en los registros de "Aprende con la lección" al mismo tiempo que el módulo "Escalas de medición". Se observó alta actividad en los mismos días, es decir, los estudiantes eligieron resolver y estudiar los dos módulos al mismo tiempo, gracias a la estructura modular de libre elección del curso. Además, es notorio que en el módulo "Descripción y organización” en el día treinta hubo 643 registros, mucho mayor que en cualquier otro módulo; esto reflejó que las visitas de las actividades pueden también estar relacionadas al tipo de programación y complejidad del contenido. Este módulo demandaba extraer, realizar cálculos, procesar datos en general y también requería de más acciones interactivas.

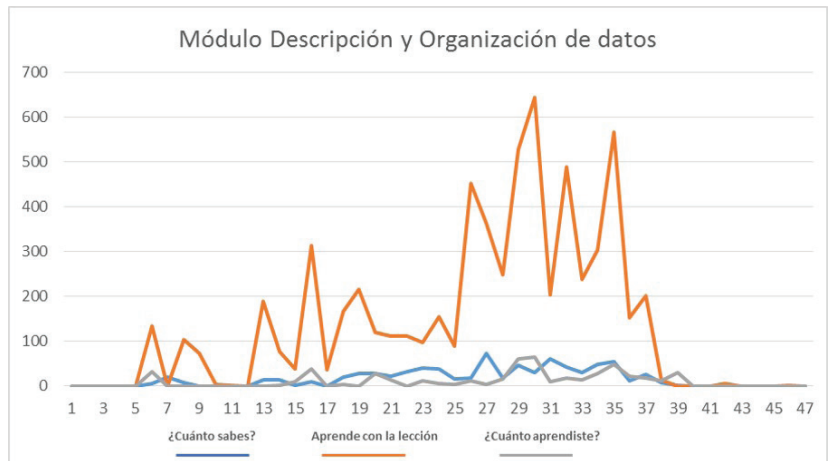

Figura 9. Registro de las actividad en el módulo Descripción y Organización.

Respecto a los resultados del módulo "Medidas de Tendencia Central" (figura 10) se registró actividad en un periodo muy marcado del día quince al día cuarenta en las tres actividades. Esto muestra que los estudiantes probablemente consideraron primero revisar contenido que les fuera recurrentes en los módulos anteriores a este. Otro aspecto importante es que los registros de actividad en la evaluación ¿Cuánto aprendiste? fueron mayores que la evaluación inicial de ¿Cuánto Sabes?, lo que denota que los estudiantes tuvieron resultados no satisfactorios en la evaluación final y debían estudiar más en la lección para volver a presentar la evaluación ¿Cuánto sabes? Esto en los módulos anteriores tuvo un patrón inverso.

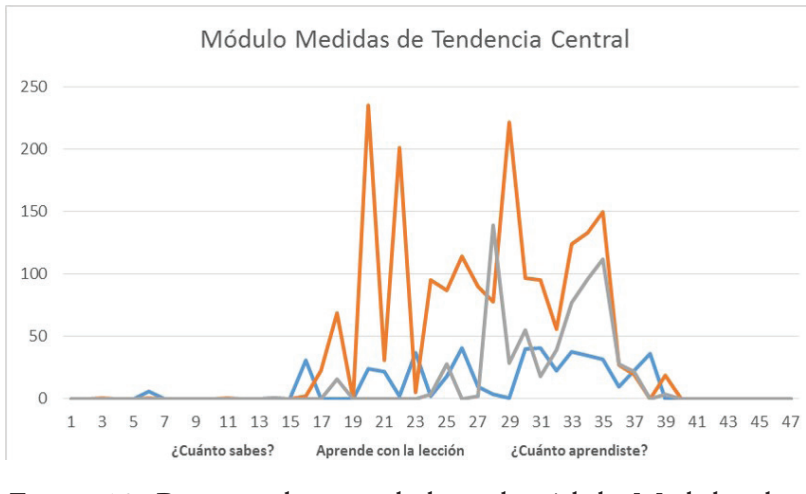

Figura 10. Registro de actividad en el módulo Medidas de Tendencia Central. 
Los registro de actividad de los módulos "Dispersión” y "Tablas y Figuras" (figuras 11 y 12) presentan ciertas similitudes, ya que en ambas el registro comienza el día seis y siete con un máximo de 29 visitas en la actividad de "Aprende con la lección” del módulo "Dispersión” y 52 en el módulo "Tablas y Figuras". Posterior a esto, las visitas se postergaron hasta el día diecisiete en el caso del módulo "Dispersión" y día dieciocho en el caso del módulo "Tablas y Figuras". Es posible que los estudiantes exploraron estos módulos en el inicio del curso, pero decidieron dejarlos y revisar los primeros módulos de "Medición" y "Variables". Cabe mencionar también que la actividad de "Aprende con la lección" del módulo "Tablas y Figuras" fue la actividad con mayor registro de actividad, con 813 visitas el día treinta y cinco, debido probablemente a que se acercaba el cierre del curso y se quería completar todos los módulos. Ambos módulos fueron dejados al final dentro de la ruta de aprendizaje, seguida por los estudiantes, pero el de Tablas y Figuras fue el último de estos dos (figura 12).

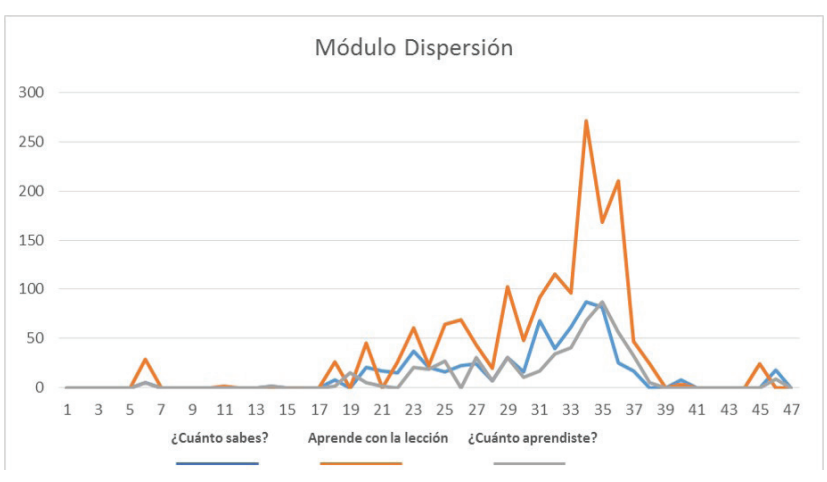

Figura 11. Registro de la actividad en el módulo Dispersión.

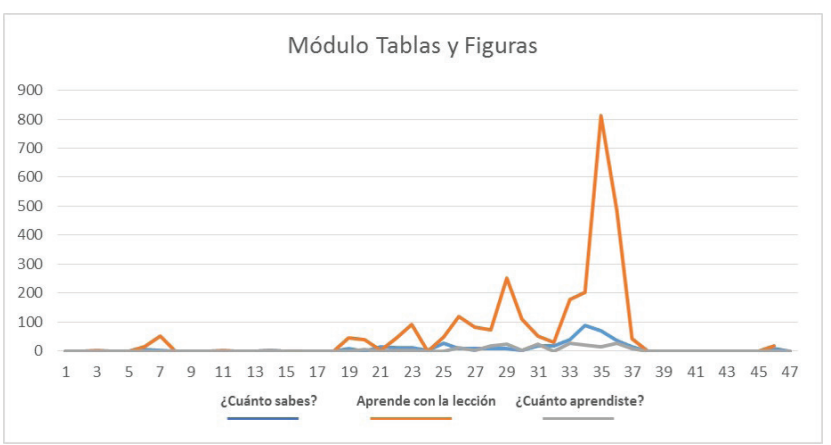

Figura 12. Registro de actividad en los módulos Tablas y figuras.
En la figura 13 se muestran los registros de los estudiantes en las evaluaciones, por cada módulo. Se observa un mayor número de ingresos a las evaluaciones de inicio ¿Cuánto sabes?, que es donde se estima el nivel de conocimientos y habilidades que poseen los estudiantes al empezar un módulo. En general, también se puede notar que los registros de actividad a cualquiera de las evaluaciones finales ¿Cuánto aprendiste? desciende de un módulo a otro, lo cual ha ocurrido de manera semejante en otros cursos online (Rivera, 2011).

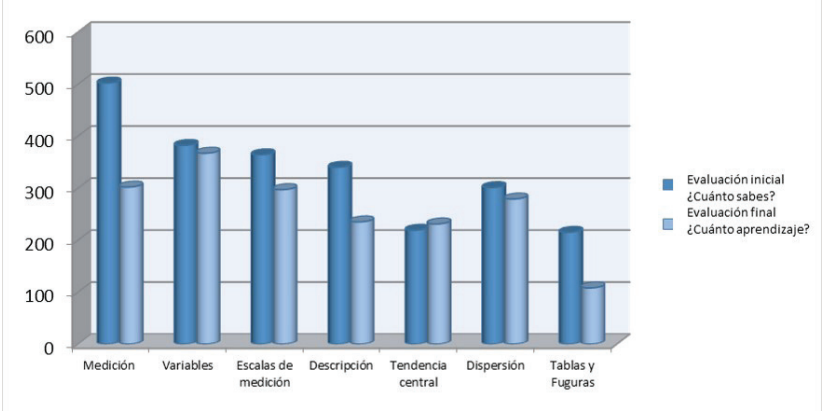

Figura 13. Registro de actividad en evaluaciones por cada módulo.

Por otra parte, en la figura 14 se muestran los registros de actividad en "Aprendiendo con la lección" de cada módulo. Aquí se puede observar una mayor frecuencia de visitas en el módulo "Descripción", que establece los aprendizajes esperados: Diferenciar el uso de las distribuciones de frecuencias, interpretar la organización de datos mediante la distribución de frecuencias, organizar una serie de datos en distribuciones de frecuencias, comprender la descripción de datos a través del porcentaje, calcular, interpretar y organizar el porcentaje en una serie de datos, diferenciar el uso entre los deciles, cuartiles y percentiles. En cambio, el módulo con menos registros de actividad fue el módulo de "Dispersión", cuyos aprendizajes esperados definidos fueron: (i) comparar cada una de las medidas de dispersión a partir de sus propiedades, seleccionar la medida de dispersión acorde al nivel de medición, la forma de su distribución y el objetivo de la investigación, (ii) interpretar los resultados de cada medida de distribución a través de la resolución 
de problemas en la investigación científica, (iii) describir el procedimiento estadístico para la obtención de cada una de las medidas de dispersión y (iv) demostrar la obtención de las medidas de dispersión mediante ejercicios.

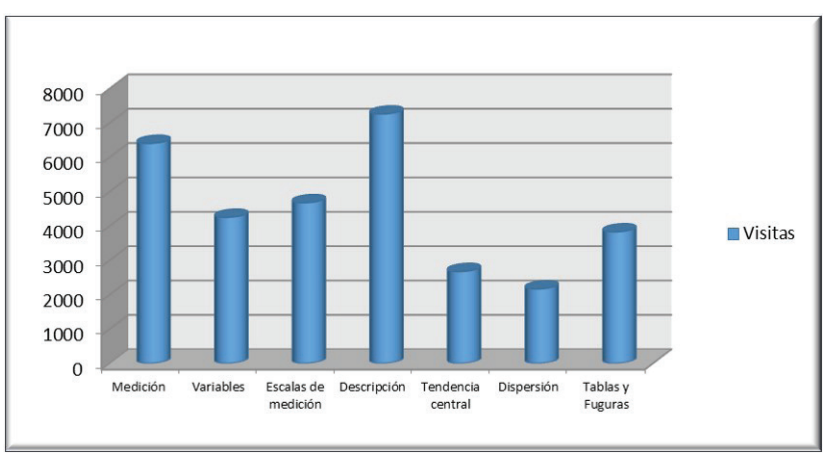

Figura 14. Registro de visitas de los estudiantes a la lección de cada módulo.

Por último, como se mencionó en la presentación del curso, éste cuenta con un espacio de interacción entre estudiantes y desarrolladores del mismo. En dicho espacio, los estudiantes inscritos en el curso autogestivo modular en línea de metodología de la investigación para universitarios, mediante comentarios compartían sus inquietudes y/o dificultades durante la navegación en la plataforma del curso y en la mayoría de los casos

se expresó satisfacción y motivación por tomar cursos de este tipo. Expresaron estar al pendiente de los siguientes cursos. Estas opiniones fueron diferentes a las obtenidas en estudios anteriores en los que se reportó que son dos los puntos de vista de los estudiantes; rechazo total a los cursos en línea en general y rechazo de cursos de matemáticas, ciencia y tecnología en línea (Ramblo, 2016).

También se reportaron dificultades durante la navegación y configuración de algunos ejercicios autoaplicables. Todos los comentarios se tomaron en cuenta en la etapa de mejoras y al hacer las adecuaciones de mejora se logró hacer más amigable y eficiente la navegación de los estudiantes en el curso.

Finalmente, según los resultados obtenidos se puede afirmar que los elementos de evaluación sumativa iniciales y finales como las lecciones de aprendizaje permitieron a los estudiantes retroalimentar su aprendizaje y tomar decisiones sobre las rutas de aprendizaje que deseaban seguir, primero explorando los módulos iniciales y finales, realizando la evaluación sumativa inicial ¿̨cuánto sabes?, después, realizando las actividades de las lecciones de aprendizaje de los primeros módulos y combinando el estudio de los módulos intermedios simultáneamente. Esto evidenció una recta de aprendizaje de secuencia no lineal como regularmente se presentan en varios cursos en línea.

\section{DisCuSión Y CONCLUSIONES}

De acuerdo a los resultados, se puede concluir que el curso autogestivo modular en línea diseñado para enseñar metodología de la investigación a universitarios permitió describir las rutas de aprendizaje de los estudiantes universitarios, quienes, independientemente de cuál sea la ruta seguida, concluyeron satisfactoriamente el curso. Se descubrió que se siguió trayectorias de aprendizaje de tipo polinómicas, lo que indica mucha actividad con periodos de incrementos o decrementos, en periodos de tres o cinco veces dentro de los 47 días que estuvo disponible el curso. Según la complejidad del módulo que estudiaban por las actividades que les demandaban, según los aprendizajes esperados del módulo. Además, esto muestra que dada la flexibilidad del curso para elegir una trayectoria según decisión del estudiante, las trayectorias politómicas indican que los estudiantes no siguieron una secuencia o ruta lineal, como en muchos cursos en línea aún lo hacen. Por ello fue posible alcanzar el objetivo de este trabajo. Esto posibilita que el curso ya pueda ser abierto de manera masiva a la comunidad universitaria, no solo de la UNAM, sino de cualquier universidad de México o de otros países.

Si bien es cierto en la actualidad las instituciones de educación superior enfrentan el reto de incorporar procesos educativos no presenciales soportados por la tecnología. Este artículo da respuesta a dicho planteamiento proponiendo el curso autogestivo modular en línea de metodología de la investigación para universitarios, con el cual se rompe con el tipo de enseñanza enciclopédica, característico en la enseñanza presencial del método científico. Esto gracias a que en el curso expues- 
to en este trabajo se retomaron en los ejercicios autoaplicables las siete dimensiones de Fossion y Zapata (2015), donde es de vital importancia tener una propuesta de investigación, diseño del estudio, planificación, recolección y procesamiento de datos, análisis de datos, conclusiones y comunicación de resultados contextualizados en los problemas que se le presentaban a los estudiantes.

Además, partiendo del supuesto antes planteado en el que se menciona que las instituciones de educación superior deben ajustarse y dar respuesta y solución a las necesidades sociales actuales. En este curso, los contenidos están ejemplificados con situaciones de investigación retomados de estudios previos y prácticas concretas, al igual que los ejercicios autoaplicables y los reactivos de las evaluaciones, tal como lo recomienda Sánchez (2014).

En otro punto, pese a que en el diseño de la plataforma de Ciencia CUVED se contempló que se fomentaría la comunicación efectiva y no solo de transmisión de información entre los estudiantes, tal como lo recomiendan Usoro \& Akuchie (2012), mediante un espacio denominado "comentarios", los estudiantes solo se enfocaron en reportar cuestiones sobre la programación de dicho curso, dejando de lado la comunicación entre estudiantes.

Ahora bien, con respecto a los sitios web revisados, donde se enseña metodología de la investigación en línea, se puede concluir que este curso, además de poseer innovadoras características pedagógicas, logró integrar contenidos teóricos y aplicados, gracias al enfoque de aprendizajes esperados, ya que los cursos mencionados con anterioridad se centraban solo en uno de estos dos aspectos, mostrando contenidos descontextualizados.

Por otra parte, con respecto al enfoque pedagógico planteado en la introducción, se puede decir que se ha comprobado cómo el aprendizaje autogestivo puesto en práctica mediante este curso es adecuado. Es posible también que influyan las habilidades de autorregulación de los estudiantes, así como sus habilidades de pensamiento, actitudes frente al estudio que pueden ser una muestra de su capacidad para ser autogestivo, autónomo o independiente (Ponce, 2016). Debido a que los estudiantes que fueron constantes en el curso lograron concluirlo de forma exitosa con una calificación superior al $60 \%$, puede verse que fueron capaces de generar su propia ruta de aprendizaje en base a sus necesidades, tal como lo recomiendan Taub et al. (2014). Además se logró un ambiente hipermedia modular que permitió a los estudiantes tomar decisiones con respecto a su aprendizaje, situarlos en un contexto de aprendizaje específico, regular su aprendizaje con las retroalimentaciones del curso y apoyar los procesos autónomos de regulación de aprendizaje mediante evaluaciones tipo examen diagnóstico, lo cual recomienda Ponce (2016) para tener un entorno de aprendizaje virtual autogestivo. Téngase en cuenta que "[...] un aspecto medular en la promoción del aprendizaje autogestivo es la flexibilidad o el control que tiene el alumno sobre los itinerarios que hace dentro del espacio tecnológico, así como de las respuestas que debe dar a las actividades"(p. 20). Un curso autogestivo con diseño flexible debe ayudar al estudiante a decidirse por una u otra ruta. Este es un elemento muy relevante, ya que estos cursos no tienen tutor y tal flexibilidad en su programación es concebida con cierta función tutorial, que conduce sutilmente al estudiante hacia los escenarios y situaciones previstas por los diseñadores. Además, por la facilidad de manipular, regresar y utilizar los videos, ejercicios, textos, multimedia, y todo elemento interactivo a libre disposición de las necesidades del estudiante, el curso materializas la idea de una educación centrada en el alumno como sujeto activo y responsable de su formación.

Por último, se recomienda para estudios futuros seguir esta línea, debido a que los ambientes virtuales no solo son un apoyo para la enseńanza presencial, sino que permiten ampliar nuevos escenarios para el aprendizaje, de sistemas presenciales, mixtos, completamente en línea e incluso informales o de corte masivo, como los mooc, pero con elementos de especialización mayor y más sofisticados para evaluar el aprendizaje a diferencia de los MOOC. 


\section{Agradecimiento}

Este artículo es un producto directo logrado gracias al financiamiento del Programa de Apoyo a Proyectos para la Innovación y Mejoramiento de la Enseñanza PAPIME de la UNAM, número PE303215: "Diseño de cursos en línea sobre estadística y manejo del paquete estadístico SPSS en su nivel básico, intermedio y avanzado para estudiantes de licenciatura y posgrado”.

\section{REFERENCIAS BIBIOGRÁFICAS}

Aguilera, C. (2005). El estudio autogestor. Guadalajara: UNIVA.

Arratia, O., Galisteo, D., Pérez M., \& García-Arista, M. (2009). Innovación en docencia universitaria con moodle. Casos prácticos. España: Club Universitario.

Atlantic Interntational University. Curso de metodología de la Investigación. [Moodle] Recuperado de: http://cursos. aiu.edu/metodologia\%20de\%20la\%20investigacion.html

Azevedo, R., Witherspoon, A., Chauncey, A., Burkett, C. \& Fike, A. (2009). MetaTutor: A MetaCognitive Tool for Enhancing Self-Regulated Learning. Department of Psychology, Institute for Intelligent Systems, Cognition and Technology Research Lab, University of Memphis, Memphis, TN, 38152, Retrieved from: https://www.aaai.org/ocs/ index.php/FSS/FSS09/paper/viewFile/995/1253

Colina, C. (2007). La investigación en la educación superior y su aplicación social. Revista de educación Laurus. 13(25). Recuperado de: http://www.redalyc.org/ pdf/761/76111479016.pdf

Duffy, M. C. \& Azevedo, R. (2015). Motivation matters: Interactions between achievement goals and agent scaffolding for self-regulated learning within an intelligent tutoring system. Computers in Human Behavior, 52, 338-348. Retrieved from:http://dx.doi.org/10.1016/j.chb.2015.05.041

Facultad de Medicina, Posgrado. (2016). Metodología de la investigación médica. [HTML]. Universidad de Navarra. Recuperado de: http://www.unav.es/posgrado/cursos-online/titulo-propio,

Fossion, R. \& Zapata-Fonseca, L. (2015). The Scientific Method. In Aging Research - Methodological Issues (pp. 9-25). Cham: Springer International Publishing. http://doi. org/10.1007/978-3-319-18323-7_2

González-Gómez, D., Jeong, J. S. \& Rodríguez, D. A. (2016). Performance and Perception in the Flipped Learning Model: An Initial Approach to Evaluate the Effective-ness of a New Teaching Methodology in a General Science Classroom. Journal of Science Education and Technology, 25(3), 450-459. DOI: $10.1007 / s 10956-016-9605-9$

Guiliano, A. \& Robertt, P. (2010). La enseñanza de las me- todologías en las ciencias sociales en Brasil. Cinta de Moebio 38: Revista de Epistemología de Ciencias Sociales. DOI: 10.4067/S0717-554X2010000200004

Guinee, K. (2012). E-learning behaviors in middle school. Encyclopedia of Cyber Behavior, I - III, 1111-1123. DOI: 10.4018/978-1-4666-0315-8.ch091

Johnson, S. K. \& Gotthard, G. H. (2015). Chapter: Using science to teach science: Applying the scientific method in teaching physiological psychology. Ed: Dana S. Dunn. The Oxford Handbook of Undergraduate Psychology Education. Oxford University Press; US, 403-418. DOI: 10.1093/oxfordhb/9780199933815.013.029

Leonard, J., Chamberlin, S., Johnson, J. B. \& Verma, G. (2016). Social justice, place, and equitable science education: Broadening urban students' opportunities to learn. The Urban Review. DOI: 10.1007/s11256-016-0358-9

Lourenço, A. B., Costa, G. G. \& Hernandes, A. (2009). Actividad de investigación científica con alumnos de nivel medio: un estudio de caso. Enseńanza de las ciencias: revista de investigación y experiencias didácticas, Extra, 2013- 2018. Recuperado de: http:/www.raco.cat/index.php/Ense- nanza/article/view/294182

Ma Watson, S. L., Loizzo, J., Watson, W. R., Mueller, C., Lim, J. \& Ertmer, P. A. (2016). Instructional design, facilitation, and perceived learning outcomes: an exploratory case study of a human trafficking MOOC for attitudinal change. Educational Technology Research and Development, 1-28. DOI: $10.1007 / \mathrm{s} 11423-016-9457-2$

Nuñez, J. C., Solano, P., González-Pienda, J. A. \& Rosario, P. (2006). Evaluación de los procesos de autorregulación mediante autoinforme. Psicothema, 18, 3, 353-358. Recuperado de: http://www.psicothema.com/pdf/3222.pdf

Park, S. Y., Kim, S. W., Cha, S. B. \& Nam, M. W. (2014). Comparing learning outcomes of video-based e-learning with face-to-face lectures of agricultural engineering courses in Korean agricultural high schools. Interactive Learning Environments, 22(4), 418-428. Retrieved from: http://dx. doi.org/10.1080/10494820.2012.680967

Peña, R. F., Waldman, F. B., de Pérez Berbain, N. M. S., Tejada, G. A., Cadirant, G. S. C., Passaglia, J. y Contrera, M. (2012). Implementación de los entornos virtuales de aprendizaje en cursos de capacitación docente. Revista Iberoamericana de educación, 60, 117-128. Recuperado de: https:// dialnet.unirioja.es/servlet/articulo? codigo $=4772494$

Peñalosa, C., E. y Castañeda-Figueras, S. (2010). Análisis cuantitativo de las modalidades interactivas en al aprendizaje en línea. Revista mexicana de investigación educativa, 15(47). Recuperado de: http://www.scielo.org.mx/scielo. php?script=sci_arttext $\&$ pid $=$ S1405-66662010000400011

Ponce, M. E. (2016). La autogestión para el aprendizaje en estudiantes de ambientes mediados por tecnología/ Self-managed learning in students within technology-mediated environments. Diálogos sobre educación, (12).

Ramlo, S. E. (2016). Students' Views About Potentially Offering Physics Courses Online. Journal of Science Edu- 
cation and Technology, 25(3), 489-496. DOI: 10.1007/ s10956-016-9608-6

Rivera-Montalvo, D. (2011). Factores que inciden en la retención o deserción del estudiante a Distancia. (Tesis doctoral inédita, Nova Southeastern University). Recuperado de: http:// ponce.inter.edu/cai/tesis/derivera/index.pdf

Rizo, M. (2010). Enseñar a investigar investigando: Experiencias de investigación en comunicación con estudiantes de la Licenciatura en Comunicación y Cultura de la Universidad Autónoma de la Ciudad de México. Universidad Autónoma de la Ciudad de México.

Recuperado de:https:// historia-sexto.wikispaces.com/file/ view/ense $\% \mathrm{C} 3 \% \mathrm{~B} 1 \mathrm{ar}+\mathrm{a}+$ investigar+investigando.+Marta+Rizo+Garc\%C3\%ADa. pdf

Rodríguez-Jiménez, R. \& Martín-López, M. (2016). Curso online de metodología de la investigación clínica. PUPILUM [HTML]. Recuperado de: https://www.pupilum. com/curso-online-de-investigacion-clinica

Rossiter, D.G. (2006). Preparation for MSc Thesis Research. ITC. Enschede, NL. Retrieved from: http://faculty.ksu.edu. sa/72563/Documents/NRM_004v7_MScResearchPreparedness.pdf

Sánchez, P. R. (2014). Enseñar a investigar: una didáctica nueva de la investigación en ciencias sociales y humanas. México: IISUE Universidad Nacional Autónoma de México Instituto de Investigaciones sobre la Universidad y la Educación México. Recuperado de: http://132.248.192.241/ - editorial/wpcontent/uploads/2014/10/Ense\%C3\%B1ar-a-investigar.pdf

Sander, B. \& Golas, M. M. (2013). HistoViewer: An interactive e-learning platform facilitating group and peer group learning. Anatomical sciences education, 6(3), 182-190. DOI: 10.1002/ase.1336

Scribano, A. Gandia, C. y Magallanes, C. (2006). La enseñanza de la "metodología de la investigación" en ciencias sociales. Ciencias sociales online. Revista Electrónica 1ll, 1. Recuperado de: http://www7.uc.cl/sw_educ/educacion/ grecia/plano/html/pdfs/linea_investigacion/Otros_IOT/ IOT_003.pdf

Silva, A. (2004). Métodos Cuantitativos en psicología. México: Trillas.

Simmons, N. (2013). Outcomes of Learning. Ed. M. Kompf \& P. Denicolo. Critical Issues in Higher Education. Volume 8 of the series Critical Issues in the Future of Learning and Teaching. USA: SensePublishers. 85-96. DOI: 10.1007/978-94-6209-046-0_7

Taub, M., Azevedo, R., Bouchet, F. \& Khosravifar, B. (2014). Can the use of cognitive and metacognitive self-regulated learning strategies be predicted by learners' levels of prior knowledge in hypermedia-learning environments? Computers in Human Behavior, 39, 356-367. Retrieved from http://www.sciencedirect.com/science/article/pii/ S0747563214003926

Tüzün, H. \& Çınar, M. (2016). Guidelines for Transferring Residential Courses into Web. The International Review of
Research In Open And Distributed Learning, 17(4). doi: http://dx.doi.org/10.19173/irrodl.v17i4.2323

Usoro, A. \& Akuchie, R. C. (2012). E-Learning in Higher Education. In Leveraging Developing Economies with the Use of Information Technology (pp. 228-237). USA: IGI Global DOI: 10.4018/978-1-4666-1637-0.ch014

Villardón, L. \& Yániz, C. (2011). Autogestión del aprendizaje y la autonomía e iniciativa personal. UNIVETS 2011. Disponible en: http://dugi-doc.udg.edu/bitstream/handle $/ 10256 / 3759 / 260$.pdf?sequence $=1$

Walter, C. (2016). What are Tutors' Experiences with Online Teaching?: A Phenomenographic Study. International Journal of Mobile and Blended Learning (IJMBL), 8(1), 1833. DOI: 10.4018/IJMBL.2016010102? 